This is a peer-reviewed, accepted author manuscript of the following article: Ramirez Marengo, C., Llobet Lopez, M., Navas de Maya, B., Giagloglou, E., Kilner, A., Kurt, R. E., Turan, O., \& Pozzi, S. (Accepted/In press). SAFEMODE's approach for incorporating human factors into risk informed design. Paper presented at 31st European Safety and Reliability Conference (ESREL) 2021, .

\title{
SAFEMODE'S APPROACH FOR INCORPORATING HUMAN FACTORS INTO RISK- INFORMED DESIGN
}

\author{
Clementina Ramirez-Marengo \\ Department of Naval Architecture, Ocean and Marine Engineering, University of Strathclyde, 100 Montrose St, Glasgow, G4 0LZ, \\ United Kingdom.E-mail: clementina.ramirez@strath.ac.uk
}

\author{
Marta Llobet Lopez \\ EUROCONTROL Experimental Centre, Centre du Bois des Bordes CS 41005, 91222 Brétigny sur Orge, France. E-mail: marta.llobet- \\ lopez@eurocontrol.int
}

Beatriz Navas De Maya

Department of Naval Architecture, Ocean and Marine Engineering, University of Strathclyde, 100 Montrose St, Glasgow, G4 0LZ, United Kingdom. E-mail: beatriz.navas-de-maya@strath.ac.uk

Evanthia Giagloglou

Department of Naval Architecture, Ocean and Marine Engineering, University of Strathclyde, 100 Montrose St, Glasgow, G4 OLZ, United Kingdom. E-mail: evanthia.giagloglou@strath.ac.uk

Andrew Kilner

EUROCONTROL Experimental Centre, Centre du Bois des Bordes CS 41005, 91222 Brétigny sur Orge, France. E-mail: andrew.kilner@eurocontrol.int

\section{Rafet Emek Kurt}

Department of Naval Architecture, Ocean and Marine Engineering, University of Strathclyde, 100 Montrose St, Glasgow, G4 0LZ, United Kingdom. E-mail: rafet.kurt@strath.ac.uk

Osman Turan

Department of Naval Architecture, Ocean and Marine Engineering, University of Strathclyde, 100 Montrose St, Glasgow, G4 0LZ, United Kingdom.E-mail: o.turan@strath.ac.uk

\section{Barry Kirwan}

EUROCONTROL Experimental Centre, Centre du Bois des Bordes CS 41005, 91222 Brétigny sur Orge, France. E-mail: barry.kirwan@eurocontrol.int

\section{Simone Pozzi}

Deep Blue, Piazza Buenos Aires 20,00198, Rome, Italy. E-mail: simone.pozzi@dblue.it

Human operators play a key role in the safe and successful conduct of maritime and aviation transport operations. Human error is often reported as a contributor to maritime and aviation accidents. Therefore, the implementation of human-informed design considerations is essential to improve safety and operational performance in both sectors, especially in the maritime sector, where there is a lack of an established framework to systematically consider human factors at the design stage. Therefore, the SAFEMODE project brings together key experts from both aviation and maritime sectors to address this important gap. The SAFEMODE project aims to deliver a framework that includes human factors considerations and enables designers to make risk-informed decisions.

The methodological approach of SAFEMODE builds upon four areas: the collection and analysis of accident data; the development of a toolkit for human performance assurance, the development of Human Factors-based risk models and the creation of a framework to support risk-informed design. The type of safety events considered in SAFEMODE for both domains includes collision and grounding for the maritime sector, and runway collision, taxiway collision and wake vortex during en-route flight phase for the aviation sector. This paper will provide an insight into the efforts conducted as part of the SAFEMODE project to assess the human contribution to risk and the benefits of applying these models to support risk-informed decisions in design and operations.

Keywords: Human Factors, Safety, Risk Assessment, Design, Maritime, Aviation. 


\section{Introduction}

The implementation of human-informed design considerations is essential to enhance safety and reduce the occurrence of incidents and accidents in the aviation and maritime sectors. Traditional approaches consider human error as a potential cause of accidents rather than analyzing the influencing factors and reasons behind these actions. Conducting a deeper analysis of the factors that contributed to an accident is very challenging due to the fact that uncovering these underlying reasons requires special knowledge and tools that are not always readily available for designers. Considering human factors in safety is complex, because it is fundamentally multi-layered in nature.

\subsection{Heinrich's Accident Pyramid}

Heinrich's accident pyramid (1931) has long been a model representing the relationship between the number of major accidents and the less severe occurrences. Throughout the years, different interpretations of the accident pyramid have been given. The accident pyramid is often used as if it describes different relationships among different categories of events (Marshall, Hirmas, and Singer 2018). This interpretation proposes that there are very large numbers of (commonly unreported) hazardous occurrences, near misses and deviations in relation to the numbers of major accidents reported. The near misses are shown as the bottom of the pyramid, which, if detected and effectively addressed, could significantly reduce the occurrence of major accidents.

Additional interpretation of the accident pyramid was later provided by Hollnagel (2014). In this interpretation, the outcomes (different categories of events) represent the cumulative effect of different causes and contributing factors. This view suggests that a limited number of causes (e.g. one thing goes wrong) correspond to less severe consequences, whereas a larger number of causes are associated with the occurrence of major accidents. Another perspective provided by the same author is that severe outcomes can be explained by the failure of preventive barriers, and that the severity of the outcomes depends on the number of barriers that have failed.

A key concept obtained from the interpretations of the accident pyramid is that in order to prevent the most severe accidents, knowledge should be gained from the less severe incidents and near misses which occur at the base of the pyramid. Therefore, underlying causes must be analyzed in order to prevent the recurrence of accidents and near misses.

\subsection{Protective barriers are not flawless}

Commonly, systems have various layers of protection that rely on instrumentation, safety barriers, people, operating procedures and administrative controls. The successful performance of protective barriers and safeguards is essential to prevent the occurrence of accidents (Hollnagel 1999). In an ideal world, the integrity of these barriers should never be compromised. However, weaknesses have been identified, proving that protective barriers are not flawless. The Swiss cheese model (Reason 2000) is an accident causation model used by different industries to represent system failures and barriers' weaknesses.

The Swiss cheese model represents barriers as slices of Swiss cheese, having many holes (barriers' weaknesses). In everyday operations, these holes are constantly moving and changing their size. The presence of holes in one or more slices does not automatically lead to an accident. An accident occurs when the holes in the different layers 'line up' to allow a hazardous situation to develop into an accident

According to this model, the holes in the barriers are due to active failures and/or the presence of latent conditions. Active failures are the unsafe acts associated with the accident. Latent conditions may lie dormant within the system before they are compounded with active failures to create an accident opportunity. Compared to active failures, latent conditions can be identified and corrected before an undesired event occurs.

\subsection{Going beyond human error}

Commonly, accidents are the result of a chain of events or sequence of barrier failures (Hollnagel 2014). Often when an accident occurs, the causes are identified, and risk control options are put in place to avoid the recurrence of the accident. This follows a "find and fix approach". In a variety of sectors, human error has been commonly identified as a cause in many incidents and accidents. However, identifying human error as a root cause of an accident is not enough to further enhance safety. Dr. Trevor Kletz, a renowned process safety expert, stated, "For a long time, people were saying that most accidents were due to human error, and this is true in a sense, but it's not very helpful. It's a bit like saying that falls are due to gravity." (Kletz 2008)

When 'human error' is identified as the cause of an accident, and there is no deeper search for contributing factors, a commonly given solution to eliminate the issue is to provide (re-)training. This is a reactive approach, and the problem may be seen as dealt with. However, if inadequate training was not the problem, safety learning has failed.

Collecting context-related information that influences human performance is therefore essential. Investigating or analyzing incidents at this level of detail will lead to more effective prevention strategies and corrective measures, and addressing systemic and structural aspects such as job design and interface design (e.g. cockpit and bridge design). For this reason, it is of paramount importance that an environment where occurrences are reported exists, and adequate resources are in place for investigation and the implementation of preventive measures. In order to create such an environment, where reporting is encouraged and 
safety learning is promoted, it is necessary to switch the focus from a blame culture to a just culture.

\subsection{Challenges for incorporating human factors in new designs}

Despite all the research that has been carried out in the past decades, there are still some challenges when addressing the contribution of human factors to transport accidents. For example, in the maritime sector, there is a lack of a suitable technique to measure the contribution of each individual human factor into maritime accidents. In addition, the incorporation of human factors into safety analysis can be seen as a complex task (Jeong et al. 2016).

With the development of new technologies and increased automation in the transportation sector, the impact of human factors on safety is likely to evolve and become even more relevant in the future. Remotely operated ships and aircraft, multicultural crews, mixed types of traffic to be managed together, and so on, are transforming human-computer interactions in different transportation modes and might introduce unknown risks (Pozzi 2020).

To assess the potential impact of new technologies and human factors in safety, the challenge lies firstly in the scarcity of human factors data obtained from the investigation of safety events, and secondly in the lack of effective feedback loops from operations back to designers (Pozzi 2020). Moreover, different approaches for analyzing and classifying human errors exist, leading to problems in consistency, harmonization and validation. Therefore, there is a need to create a framework that provides a standardized approach for data collection and a set of tools and methods that will support designers to make human risk-informed decisions.

\section{The SAFEMODE Project}

The main objective of the SAFEMODE project is to develop a novel Human Risk-Informed Design (HURID) Framework in order to identify, collect and assess human factors data. The SAFEMODE project brings together two transportation sectors in order to combine and enhance existing approaches. In this project, the maritime and aviation domains join their human factors and safety approaches in order to learn from one sector to another. SAFEMODE's vision is to capture human factors and human element aspects and their interaction with other components of the systems, in order to enhance safety in maritime and aviation operations (SAFEMODE 2020g, 2019a)

This will be achieved by:

- $\quad$ providing a cross-industry approach to capture and analyze safety events

- developing risk models that allow human actions to be understood in relation to a safety incident

- $\quad$ identifying key performance influencing factors that are relevant for both sectors
- creating a framework for Human Factors risks which will allow the quantitative assessment of the human contribution to failure and success

- developing tools that will support designers in understanding the potential impact of their design on human performance

\subsection{The Human Factors Iceberg}

Most accident investigations typically identify the immediate triggers or causes of an incident such as the wrong execution of a procedure or inadequate use of equipment (Antonsen 2009). The analysis focus at this level is observable performance, and many investigations fail to address the underlying causes of these conditions. There is usually little, or no design insight, and the reasons why it happened are not further investigated, which increases the possibility of the event recurring. Commonly, safety improvements are expected to be achieved by training crew members to deal with the limitations of inadequately designed systems, which often succeeds in limiting the costs but not in permanently improving the safety level. This 'surface layer', typically observed in incident reports, is only the visible tip of the iceberg. Usually, there is much more underneath. The underlying layers are where most of the safety learning can be generated. Therefore, to prevent the recurrence of an incident, attention should be given to what is under the waterline, and not only focus on what is on the surface. One of the main challenges of the SAFEMODE project is decoding the different layers of the human factors iceberg (SAFEMODE 2020g; Pozzi 2020).

In the human factors iceberg, the second layer represents human performance (Figure 1). Here, a range of factors influencing performance are considered, which include various human factors concepts such as workload, vigilance, communication, stress, teamwork, fatigue and situation awareness. These factors are elements of the Human Performance Envelope (HPE). The concept of Human Performance Envelope (HPE) suggests that when studying human performance and recovery, it is necessary to consider the different factors that can influence performance and identify which of these factors are moving outside tolerable levels. When factors affecting performance remain within tolerable limits, accidents are less likely to occur (Edwards et al. 2010; Graziani et al. 2016; Silvagni et al. 2015).

Factors affecting human performance are often used to quantify human reliability (Human Reliability Assessment), as well as those that can be used for more qualitative approaches such as Human Factors Assurance Levels (HALs: (Mana, De Rede, and Fowler 2007) and Human Performance Standards of Excellence (EUROCONTROL 2015). The analysis of incidents and accidents at this level will lead to the creation of more effective prevention strategies. The analysis focus is on the interactions between system elements, using standard Human Factors methods such as the Human Factors Analysis and Classification System (HFACS) taxonomy (which describes four levels of failure within Reason's model: unsafe acts, preconditions 
for unsafe acts, unsafe supervision and organizational influences) (Shappell and Wiegmann 2000) and the Software, Hardware, Environment, Liveware (SHELL) model used to analyze the interaction of multiple system components: software, hardware, environment and liveware (ICAO 2012; Skybrary), or task analysis techniques.

The third layer concerns the need to understand how tasks are performed (work as done) rather than assuming that operators follow procedures and training rigorously (work as imagined or as intended).

Variability is an important factor to take into account when assessing the performance of systems. Variability is an intrinsic characteristic of real systems, especially in aviation and maritime transport operations where weather and other factors play a role, and people adapt accordingly. Therefore, taking into account performance variability and learning from everyday operations is essential to improve safety.

Meeting targets in a time and resource-pressured transportation industry places high demands on operators, which may result in workarounds. This situation can allow new risks to emerge. Therefore, in situations where successful human performance is key to ensure the safety of operations, determining how tasks are performed becomes of great importance. The issue with ignoring this layer is that any attempt to correct an existing problem may not succeed if it relates to the formal prescription of work, rather than the way in which the task is conducted. Therefore, the third layer represents the 'Safety II' paradigm (EUROCONTROL 2013; Hollnagel 2014).



Fig. 1. The Human Factors' Iceberg adapted from SAFEMODE (2020g)

The final layer of the iceberg considers the culture of each sector, which reflects individual, group and organizational attitudes and behaviours. Culture has been identified as an underlying cause of accidents across different sectors (Mathis 2013; Antonsen 2009). Three levels of cultures have relevance to safety management initiatives: professional, organizational and national (ICAO 2012).

Professional culture reflects the characteristics and values of particular professional groups. Usually, professional culture is dominant, e.g. for pilots, controllers, ship's captains or engineers.

Organizational culture consists of the characteristics and values of organizations e.g. the behaviour of members of one company versus that of another company. Organizational culture, for example, reflects how much the company focuses on safety, as well as its level of just culture, which can have a great impact on safety performance (Skybrary 2020; ICAO 2012).

National culture can also play a role. National culture reflects the national characteristics and value systems of nations. For example, the response of people to authority or the way they deal with uncertain situations may differ among people of different nationalities (Hofstede 2001)

How safety culture is being embraced by designers and developers who are creating and operating new safetycritical technologies is a key question that SAFEMODE will address. Another key question relates to the differences between the aviation and maritime safety cultures, with potentially differing implications for each transport mode. The understanding of this layer will be essential to ensure that the implementation of the proposed safety solutions is sustainable.

\subsection{The SAFEMODE approach}

The SAFEMODE methodological approach follows three steps (Figure 2).

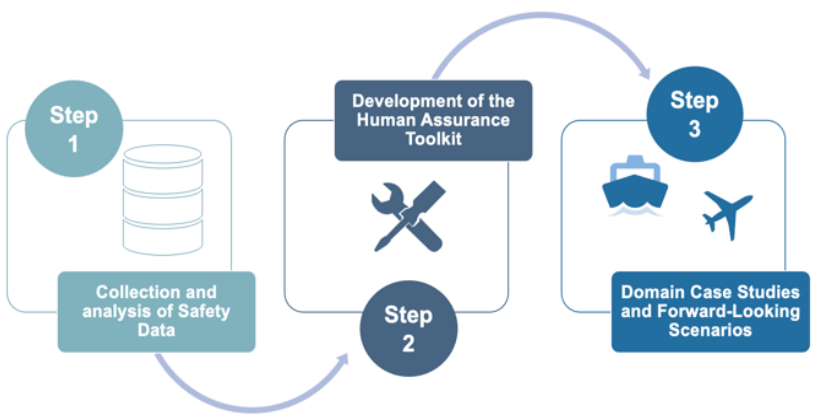

Fig. 2. SAFEMODE's methodological approach adapted from SAFEMODE (2019a)

\subsubsection{Development of the SHIELD Open Data Repository}

In this step, data on human performance in safety-relevant situations such as safety occurrence reports will be gathered. To structure and analyze the data, SAFEMODE developed a Human Factors taxonomy, which is the core of the database design, describing the contributing factors and their relationships (SAFEMODE 2019b, 2020b). 
The type of data collected include:

- occurrence facts such as location, date, injuries, type of vehicle/vessel, actors involved, and contextual conditions

- occurrence assessment, which describes the human factors that contributed to the occurrence

- $\quad$ safety positive actions and learning, which provide the actions of human operators and/or technical systems that prevented occurrences or mitigated the consequences

- the lessons learnt from the accident

- the lessons learnt from safety events

The data will help inform risk-based design of systems and operations related to the aviation and maritime sectors. The SAFEMODE project intends to overcome the unavailability of systematically collected data and the lack of collaboration between different transports models.

\subsubsection{Development of a toolkit for Human Assurance}

The aim of SAFEMODE is to provide tools, which designers and risk assessors with different levels of human factors expertise can use for their activities, addressing gaps in current design and operational processes. Therefore, the next step is to develop human risk-based tools that can be used by designers, safety professionals, or developers to make risk-informed decisions on new procedures or designs. The Human Factors Toolkit includes a large variety of methods that can be used for different purposes in the context of designing/redesigning a safety-critical system or its operations (SAFEMODE 2020c).

The development of the toolkit involves three phases:

Phase I: In recent years, there has been a growing body of research into human factors topics. A state-of-the-art review of the knowledge generated by the human factors community has been conducted. This review focused on existing human factors methods and tools in order to identify the best and the most commonly adopted by industry.

Phase II: It is anticipated that the HURID Framework will be used differently by different end-users. The HURID Use Cases describe how the HURID Framework can be utilized by various end-users to inform design decisions. During the second phase of the development of the toolkit, the identified human factors methods will be mapped onto the HURID Use Cases, evaluating their suitability for SAFEMODE.

Phase III: During this phase, the selected methods will be customized with the support of end-users to be adopted by SAFEMODE and integrated into the Toolkit.

In the HF Toolkit, the techniques have been classified into seven different clusters, depending on the specific type of service they are offering to various end-users in the context of a design process (SAFEMODE 2020c). Figure 3 shows this classification.

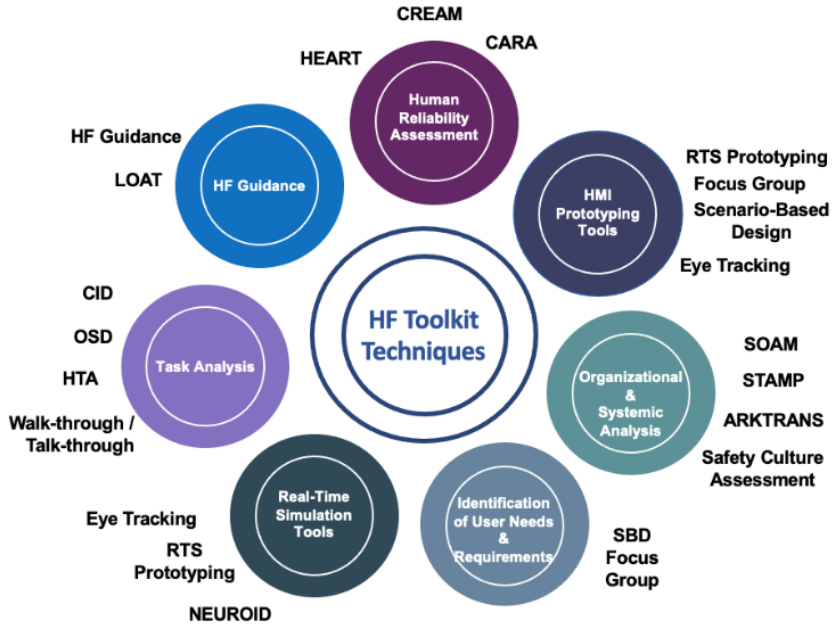

Fig. 3. Clusters of services offered by the techniques adapted from SAFEMODE (2020f)

Each human factors tool is described using a common format, along with an illustrative maritime and aviation example. Later on, in SAFEMODE, guidance will be available to help users (e.g. designers) determine which tools to use, according to design maturity, resources available, and other criteria.

The human factors methods are not expected to be used in isolation but in combination with the other components of the HURID Framework, including the SHIELD Database and the human factors-based risk models.

\subsubsection{Use of Quantitative Risk Models to Assess Risk}

Although a new design does not have to be focused on safety, it is necessary to ensure that it does not introduce new risks. A quantitative risk model can be applied to assess the contribution to the risk of the design change. As part of the SAFEMODE project, risk models for both the aviation and maritime domains will be developed. Each risk model is for a specific type of occurrence, in a specific operational context, and taking into account specific services and systems preventing or contributing to the risk of the accident. The type of accidents considered in SAFEMODE for both domains includes collision and grounding for the maritime sector, and runway collision, taxiway collision and wake vortex during en-route fligh phase for the aviation sector.

The risk models are barrier-based and allow the factors contributing to risk and key barriers in each domain to be described. Three main parts compose this risk framework: backbone, contributors and influence layer (Figure 4).

The backbone is the main part of the structure of the risk model. The backbone is mainly composed of a set of precursors, which are safety events or hazards, and a set of barriers potentially preventing them. The top of the backbone corresponds to the occurrence. The different 
elements in the backbone are linked in the form of a barrier model, equivalent to the "Swiss Cheese" metaphor, describing the layers of protection provided in each operation, and are represented as a sequence of barriers against each accident type.

The next level in the risk models is provided by the contributors. They correspond to the causal factors contributing to the execution, effectiveness and/or occurrence of the main elements of the backbone (barriers and induced events).

The final level in the risk model is the influence layer. This layer consists of a set of additional base events attached to the lowest level components in each barrier that describe a human-related event. The influence layer provides more information on factors that contributed to the human failure event (SAFEMODE 2020d).

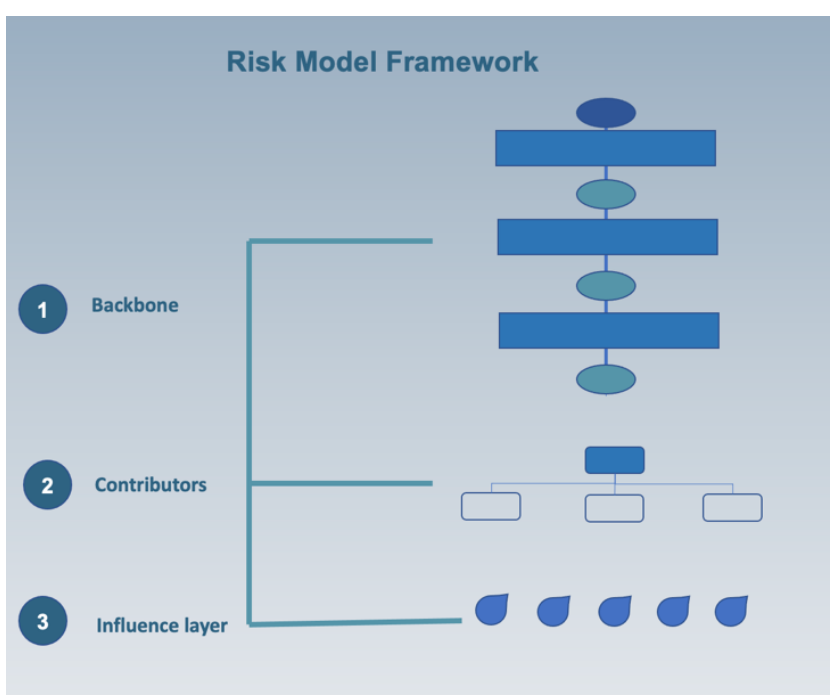

Fig. 4. SAFEMODE's Risk Models Framework adapted from SAFEMODE (2020d)

The risk models for both domains describe how a failure in a base event can lead to a major accident. Each reportable operational occurrence begins with action or failure that affects the effective performance of a barrier. Subsequent actions are taken by the operators, which, in combination with equipment and procedure failures, result in a 'top event'. The top event is the most serious occurrence. For example, in the case of aviation, this might be a mid-air collision, while in the maritime domain, it might be a collision between two vessels. In addition to identifying the causes of failure, it is essential to capture the successful human interventions that contributed to preventing an occurrence from developing further into an accident. Therefore, events described in the risk model have the capacity to represent either a failure or a success. Incident reports are used as the source to determine which events have contributed directly to failure and which have contributed directly to success (SAFEMODE 2020e).

New designs with low maturity represent another case where the evaluation of risk is required. One example in the aviation sector is the design of an AI system to support remote tower controllers in very busy periods. This system should help reduce risk but might need additional safety requirements to ensure that risk could not be increased in particular circumstances. An example in the maritime sector is a collision-avoidance system to support decision-making and reduce the risk of collision. However, extra assurances will be required in order to avoid the introduction of new risks. In both cases (aviation and maritime) the design maturity is low given that we don't yet have such support systems; therefore, formal risk modelling may not be possible. However, qualitative risk evaluation approaches such as Hazard and Operability Study (HAZOP)(Kletz 1992) and System-Theoretic Accident Model (STAMP) (Leveson 2016) could be used to identify the potential risk and determine safety requirements.

\subsubsection{Domain Case Studies and Forward-Looking Scenarios}

The HURID Framework follows a multi-step approach, which includes three major components to support the designer: the SHIELD database, the Human Factors Toolkit and domain-specific risk models (SAFEMODE 2020f). In HURID, these elements can be used separately or combined to support risk-informed decision-making by considering human factors.

Domain Case Studies will complement the developed datadriven approach, in order to address three key challenges of incident data collection:

- the level of detailed human factors information provided in accident and incident reports

- the scarcity of data on extreme cases, which occur very rarely

- availability of data for unknown risks

To complement the collected information, SAFEMODE will gather additional data, use the simulator to generate data for specific cases and develop a set of dedicated forward-looking scenarios (SAFEMODE 2020a).

In addition to supporting data collection, the Domain Case Studies will help assess how HURID can support riskinformed design and operations (SAFEMODE 2021a, 2021b). The validation of the HURID Framework will be conducted in two stages. The first stage involves the collection of feedback from different target end-users. The second stage of the validation focuses on demonstrating the applicability of the different elements of the HURID Framework in the Domain Case Studies. For each domain, three case studies were developed.

The HURID tools and methods applied in the Domain Case Studies vary depending on the scenarios under study. For example, the selection of these tools and methods may be 
based on the maturity of the design. The majority of the Case Studies incorporate various human factors techniques such as prototyping sessions that integrate several HMI design guidelines, risk models and real-time simulations.

The final outcome of this approach is the creation and validation of the HURID framework to support riskinformed design and operation by addressing a set of human factors and design-related questions (Figure 5).

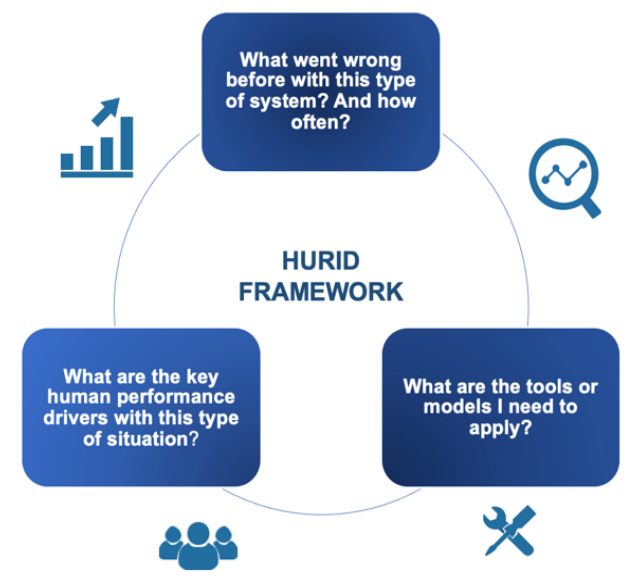

Fig. 5. Application of the HURID Framework adapted from SAFEMODE (2020g)

\section{Summary and Conclusions}

In the transport industry, addressing human factors and enhancing safety in design and operations is of paramount importance. SAFEMODE has taken on the challenge of integrating the SHIELD database and taxonomy, the human factors toolkit and domain-specific risk models into one framework to support designers in the development of new systems or procedures. This paper has presented the approach followed in the SAFEMODE project to develop the HURID Framework in order to identify, collect and assess human factors data. HURID will help designers to understand the potential impact of their design on human performance and risk. Moreover, the HURID Framework can be utilized at various stages of the design, from the early concept stage to the detailed design prior to deployment.

Due to this collaboration between the aviation and maritime sectors, the outcomes of the SAFEMODE project will provide a cross-industry approach to capture and analyze safety events. In addition, the collection and analysis of incident data within SAFEMODE will allow the identification of the key performance influencing factors that are relevant for both the aviation and maritime sectors. The Domain Case Studies aim at supporting the validation of HURID by applying its multi-step approach to the development of new operational concepts. For aviation domain the following scenarios will be used to validate HURID: En-route wake vortex alert, drone-related forwardlooking scenario and intelligient planning tool for multiple remote tower concept; whilst for the maritime domain, the following will be used: operator assessment of remotecontrol ships, implementation of a human factors and risk- based investigation suite for maritime end users and simulator-based evaluation of human response in emergencies.

Through the validation, it will also be demonstrated that the necessary human factor elements are satisfactorily considered to ensure successful human performance during the operation of the new concepts or systems. Furthermore, the creation of domain-specific risk models will support the assessment of the human contribution to risk while considering the actors and systems that are specific for each domain. The next steps in SAFEMODE include the consolidation of the HURID Use Cases based on the interaction with end-users, the customization of the methods and tools in HURID and the validation of HURID with the aviation and maritime Domain Case Studies. This will ensure that HURID is sustainable and can be utilized by organizations of various sizes and professionals with different levels of human factors expertise. The application of the HURID Framework will support design through the implementation of various human factors activities:

- identification of the human factors issues to be addressed, which will be supported by accident analysis

- identification of the human factors areas which will be affected by new designs

- assessment of the impact that new designs (concepts or procedures) will have on human performance

- analysis of human factors, which will aim at characterizing the interactions among the different actors and systems involved

- development of human factors specifications such as new roles and responsibilities, new HMI, new tasks and required skills and training.

Moreover, the application of HURID in the Domain-Case studies will demonstrate the suitability of the Framework to support human risk-informed design in realistic and practical contexts. Ultimately, the approach created in SAFEMODE will allow designers and developers to evaluate the impact of design changes on overall risk, built on a firm understanding of the critical human factors associated with the design. The benefits of incorporating human factors into the design of systems and operations will be improved safety assurance, more effective and resilient operations, and enhanced human performance. The outcomes from SAFEMODE project can also help other sectors who would benefit from the application of a framework to incorporate human factors into new designs. Transfebility to other sectors will require further investigation.

\section{Acknowledgement}

This publication is based on work performed in SAFEMODE which has received funding from the European Union's Horizon 2020 research and innovation programme under grant agreement No 814961. Any 
dissemination reflects the author's view only and the European Commission is not responsible for any use that may be made of information it contains.

\section{References}

Antonsen, S. 2009. Safety culture : theory, method and improvement. Farnham: Farnham : Ashgate Pub.

Edwards, T., S. Sharples, J.R. Wilson, and B. Kirwan. 2010. "The need for a multi-factorial model of safe human performance in air traffic control." 28th Annual European Conference on Cognitivie Ergonomics, Delt: The Netherlands, 25th-27th August.

EUROCONTROL. 2013. From Safety-I to Safety-II: A White Paper.

---. 2015. A EUROCONTROL-FAA Action Plan 15 White Paper.

Graziani, I., B. Berberian, B. Kirwan, P. Le Blaye, L Rognin, and S. Silvagni. 2016. "Development of the Human Performance Envelope Concept for Cockpit HMI Design." HCI-Aero 2016 International Conference on Human-Computer Interaction in Aerospace, Paris, France.

Heinrich, H.W. 1931. Industrial accident prevention: A scientific approach.

Hofstede, G. 2001. Culture's consequences : comparing values, behaviors, institutions, and organizations across nations. 2nd ed.. ed. Thousand Oaks, Calif.: Thousand Oaks, Calif. : Sage Publications.

Hollnagel, E. 1999. "Accidents and barriers." Cognitive Science Approaches to process Control.

---. 2014. Safety-I and safety-II: the past and future of safety management. Farnham : Ashgate.

ICAO. 2012. Safety Management Manual (SMM).

Jeong, K., B. Choi, J. Moon, D. Hyun, J. Lee, I. Kim, G. Kim, and S. Kang. 2016. "Risk assessment on abnormal accidents from human errors during decommissioning of nuclear facilities." Annals of Nuclear Energy 87: 16.

Kletz, T. 1992. Hazop and hazan : identifying and assessing process industry hazards. 3rd ed.

---. 2008. In Anatomy of a disaster, edited by CSB.

Leveson, N. G. 2016. Engineering a Safer World : Systems Thinking Applied to Safety. The MIT Press.

Mana, P., J. M. De Rede, and D. Fowler. 2007. Assurance levels for ATM elements: human (HAL), operational procedure (PAL), software. Stevenage: Stevenage: IET.

Marshall, P., A. Hirmas, and M. Singer. 2018. "Heinrich's pyramid and occupational safety: A statistical validation methodology." Safety Science 101: 180-189. https://www.sciencedirect.com/science/article/pii/S092 5753517305222 .

Mathis, T. L. 2013. Steps to safety culture excellence Edited by Shawn M. Galloway. New York: New York : Wiley.

Pozzi, S. 2020. "Human Factors towards Increasing Automation \& Autonomy." 10th EASN Conference.

Reason, J. 2000. "Human error: models and management." BMJ (Clinical research ed.) 320 (7237): 768-770. https://doi.org/10.1136/bmj.320.7237.768. https://pubmed.ncbi.nlm.nih.gov/10720363 https://www.ncbi.nlm.nih.gov/pmc/articles/PMC1117770/. SAFEMODE. 2019a. "About SAFEMODE." Accessed March 17. http://www.safemodeproject.eu/about-safemode.
---. 2019b. SAFEMODE D2.1: SHIELD human factors taxonomy for risk analysis.

---. 2020a. Newsletter 1: Project in a nutshell. http://www.safemodeproject.eu/uploadFile/142020142 2178022689.pdf.

---. 2020b. Newsletter 2: SAFEMODE in progress. http://www.safemodeproject.eu/uploadFile/312202010 55527458606.pdf.

---. 2020c. SAFEMODE D3.1: Human Assurance Toolkit and Guidance.

---. 2020d. SAFEMODE D4.1: Risk framework (methodology) for the development of different safety models incorporating Human Factors in both transport modes.

---. 2020e. SAFEMODE D4.2: Risk models of major accident types in both domains.

---. 2020f. SAFEMODE D5.1 HURID Use Cases and HURID First Release.

---. 2020g. SAFEMODE: Strengthening synergies between Aviation and maritime in the area of human Factors towards achieving more efficient and resilient MODE oftransportation. http://www.safemodeproject.eu/uploadFile/166202010 54515924907.pdf.

---. 2021a. SAFEMODE D6.2 Validation Plan: CONOPS, scenarios, technical equipment, experimental design.

---. 2021b. SAFEMODE D7.2 Validation Plan: CONOPS, scenarios, technical equipment, experimental design.

Shappell, S.A., and D.A. Wiegmann. 2000. The Human Factors Analysis and Classification System-HFACS. U.S. Department of Transportation.

Silvagni, S., L. Napoletano, L. Graziani, P. Le Blaye, and L. Rognin. 2015. Concept for Human Performance Envelope.

Skybrary. "ICAO SHELL Model." Accessed March 17. https://www.skybrary.aero/index.php/ICAO_SHELL_ Model.

---. 2020. "Organisational Culture." Last Modified 2020. Accessed March 17. https://www.skybrary.aero/index.php/Organisational C ulture. 